\title{
Full NLO electroweak corrections to Z-boson pair production at the Large Hadron Collider
}

\author{
Benedikt Biedermann* \\ Universität Würzburg, Institut für Theoretische Physik und Astrophysik, Emil-Hilb-Weg 22, \\ 97074 Würzburg, Germany \\ E-mail: benedikt.biedermmann@physik.uni-wuerzburg.de
}

\begin{abstract}
We report on a recent calculation of the full next-to-leading-order electroweak corrections to Z-boson pair production with subsequent decays into four charged leptons. Using the complete matrix elements at leading order and next-to-leading order in the electroweak coupling for the processes $\mathrm{pp} \rightarrow \mu^{+} \mu^{-} \mathrm{e}^{+} \mathrm{e}^{-}$and $\mathrm{pp} \rightarrow \mu^{+} \mu^{-} \mu^{+} \mu^{-}$, this includes all off-shell effects of intermediate massive vector bosons and photons. We employ a gauge-invariant splitting for the electroweak corrections into purely weak and photonic corrections. The latter show the well-known radiative tails near kinematical thresholds or resonances. The former are generically at the level of $\sim-5 \%$ for the fiducial cross section and reach several $-10 \%$ in the high-energy tails of distributions due to logarithms of electroweak origin. The impact of interference effects due to equal-flavour leptons in the final state can reach the order of $5 \%$ in off-shell-sensitive regions. Photon-induced contributions are included in our calculation, but turn out to be phenomenologically unimportant.
\end{abstract}

XXV International Workshop on Deep-Inelastic Scattering and Related Subjects 3-7 April 2017

University of Birmingham, $U K$

\footnotetext{
* Speaker.
} 


\section{Introduction}

Vector-boson pair production represents an important class of processes at the Large Hadron Collider (LHC). Due to their sensitivity to the triple gauge-boson couplings these processes are sensitive to effects from physics beyond the standard model. Moreover, they constitute an important background to the decay of the Higgs-boson into weak gauge-boson pairs. Most of the theoretical predictions for this process class focused so far on QCD corrections which are meanwhile known at next-to-next-to-leading order (NNLO) accuracy in the strong coupling $[1,2,3]$. The next-toleading order (NLO) electroweak (EW) corrections to vector-boson pair production are known in the literature for on-shell $\mathrm{W}$ and $\mathrm{Z}$ bosons [4, 5]. For ZZ and WW production, the full NLO EW corrections that fully take into account all off-shell effects have been presented recently $[6,7,8,9]$. In this proceedings contribution, we follow Ref. [8] and focus on the full EW corrections to the production of Z-boson pairs with subsequent decays to four charged leptons. Using complete matrix elements for the hadronic processes $\mathrm{pp} \rightarrow \mu^{+} \mu^{-} \mathrm{e}^{+} \mathrm{e}^{-}$and $\mathrm{pp} \rightarrow \mu^{+} \mu^{-} \mu^{+} \mu^{-}$, this includes all off-shell effects of intermediate massive vector bosons and photons.

\section{Numerical setup}

The leading-order (LO) contribution constitutes of the quark-antiquark annihilation channels $\bar{q} q / q \bar{q} \rightarrow \mu^{+} \mu^{-} \mathrm{e}^{+} \mathrm{e}^{-}, \mu^{+} \mu^{-} \mu^{+} \mu^{-}$at order $O\left(\alpha^{4}\right)$ with massless quark flavours $q=\{\mathrm{u}, \mathrm{d}, \mathrm{c}, \mathrm{s}, \mathrm{b}\}$. The NLO EW corrections at order $O\left(\alpha^{5}\right)$ comprise virtual and real contributions of the $\bar{q} q$ channels, $\bar{q} q / q \bar{q} \rightarrow \mu^{+} \mu^{-} \mathrm{e}^{+} \mathrm{e}^{-}(+\gamma), \mu^{+} \mu^{-} \mu^{+} \mu^{-}(+\gamma)$, and the real photon-induced contributions with one (anti)quark and one photon in the initial state. The latter, generically referred to as $q \gamma$ channels in the following, are obtained from the $\bar{q} q$ channel by crossing the real final-state photon with an initial-state (anti)quark. The one-loop corrections are further split in a gauge-invariant way into a purely weak part and a photonic contribution. The photonic part is defined as the collection of all diagrams with at least one photon in the loop coupling to the external fermion lines. The purely weak part consists of the remaining one-loop diagrams. We remark that the IR divergences in the photonic part of the virtual corrections are entirely cancelled by the corresponding divergences of the real corrections that we isolate via the dipole subtraction formalism $[10,11]$. The purely weak NLO corrections are thus by construction IR finite with LO kinematics. We have also computed the purely photon-induced contribution $\gamma \gamma \rightarrow \mu^{+} \mu^{-} \mathrm{e}^{+} \mathrm{e}^{-}, \mu^{+} \mu^{-} \mu^{+} \mu^{-}$. As this channel turns out to be strongly suppressed with respect to the $\mathrm{LO}$ cross section, we do not compute any corrections to this contribution.

The complex-mass scheme [15] has been used for consistently treating the massive gaugeboson resonances. The matrix elements have been computed with RECOLA [12] in combination with the tensor-integral library COLLIER [13], and cross checked against amplitudes from the private MATHEMATICA package POLE [14] and against a private implementation based on diagrammatic methods like those developed for four-fermion production in electron-positron collisions [15].

In the analysis presented below, we consider the LHC at a centre-of-mass (CM) energy of $13 \mathrm{TeV}$. The employed input parameters (masses and widths of the particles and coupling constants) can be found in Ref. [8]. As parton distribution function, we use the NNPDF-2.3 NLO set 


\begin{tabular}{|l|r|cccc|}
\hline & \multicolumn{1}{|c|}{$\sigma_{\bar{q} q}^{\mathrm{LO}}[\mathrm{fb}]$} & $\delta_{\bar{q} q}^{\text {weak }}(\%)$ & $\delta_{\bar{q} q}^{\text {phot }}(\%)$ & $\delta_{\gamma \gamma}(\%)$ & $\delta_{q \gamma}(\%)$ \\
\hline \hline$[2 \mu 2 \mathrm{e}]$ & $11.4962(4)$ & -4.32 & -0.93 & +0.13 & +0.02 \\
\hline$[4 \mu]$ & $5.7308(3)$ & -4.32 & -0.94 & +0.11 & +0.02 \\
\hline
\end{tabular}

Table 1: LO cross sections for $\mathrm{pp} \rightarrow \mu^{+} \mu^{-} \mathrm{e}^{+} \mathrm{e}^{-}+X$ and $\mathrm{pp} \rightarrow \mu^{+} \mu^{-} \mu^{+} \mu^{-}+X$ with the relative corrections $\delta_{i}=\sigma_{i} / \sigma_{\bar{q} q}^{\mathrm{LO}}$ for the LHC at $\sqrt{s}=13 \mathrm{TeV}$.

including QED corrections with $\alpha_{\mathrm{s}}\left(M_{\mathrm{Z}}\right)=0.118$ [16]. We consider a minimal set of selection cuts restricting the charged leptons $\ell_{i}$ in transverse momentum $p_{\mathrm{T}, \ell_{i}}$, rapidity $y_{\ell_{i}}$ and lepton separation $\Delta R_{\ell_{i}, \ell_{j}}$ according to

$$
p_{\mathrm{T}, \ell_{i}}>p_{\mathrm{T}, \min }=15 \mathrm{GeV}, \quad\left|y_{\ell_{i}}\right|<2.5, \quad \Delta R_{\ell_{i}, \ell_{j}}=\sqrt{\left(y_{i}-y_{j}\right)^{2}+\left(\Delta \phi_{i j}\right)^{2}}>0.2 .
$$

Photons from real Bremsstrahlung are recombined with the closest charged lepton if their separation obeys $\Delta R_{\ell_{i}, \gamma}<0.2$.

\section{Phenomenological results}

The results for the fiducial cross section in the setup described in the previous section are presented in Tab. 1 both for the mixed-flavour $[2 \mu 2 \mathrm{e}]$ and equal-flavour $[4 \mu]$ final state. The first column gives the absolute prediction at LO. The equal-flavour case is approximately a factor of two smaller than the mixed-flavour case. Since the selection cuts described above are symmetric under exchange of the final-state leptons, the deviation from a naive symmetry factor of two can be directly attributed to interference terms that are only present in the equal-flavour case. From the ratio $\sigma_{\mathrm{LO}}[2 \mu 2 \mathrm{e}] /\left(2 \sigma_{\mathrm{LO}}[4 \mu]\right) \approx 1.003$ we find a negative interference of about $0.3 \%$ for the fiducial LO cross section.

The four columns on the right-hand side of Tab. 1 state the relative NLO EW corrections to the $\bar{q} q$ contribution and the relative contributions of the photon-induced channels $\delta_{\gamma \gamma}=\sigma_{\gamma \gamma} / \sigma_{\bar{q} q}^{\mathrm{LO}}$ and $\delta_{q \gamma}=\sigma_{q \gamma} / \sigma_{\bar{q} q}^{\mathrm{LO}}$. The $\bar{q} q$ contribution $\delta_{\bar{q} q}^{\mathrm{EW}}=\Delta \sigma_{\bar{q} q}^{\mathrm{EW}} / \sigma_{\bar{q} q}^{\mathrm{LO}}$ is split into the purely weak and the photonic part, $\delta_{\bar{q} q}^{\text {weak }}$ and $\delta_{\bar{q} q}^{\text {phot }}$, respectively, so that $\delta_{\bar{q} q}^{\mathrm{EW}}=\delta_{\bar{q} q}^{\mathrm{weak}}+\delta_{\bar{q} q}^{\text {phot }}$. The relative corrections are at the level of the fiducial cross section equal for the mixed-flavour and the equal-flavour final state up to corrections at the sub-permille level. While this holds in the rather inclusive setup described in the previous section, this is not necessarily the case in other phase-space scenarios as has been pointed out in Ref. [8] for a setup with asymmetric selection cuts and a fiducial volume that enhances off-shell effects.

The bulk of the NLO corrections stems from the $\bar{q} q$ contribution with $-5.3 \%$ which is dominated by the purley weak contribution. The impact of the purely photon-induced contribution $\delta_{\gamma \gamma}$ matters only at permille level. The contribution is suppressed via the small photon flux in the proton. Furthermore, in contrast to the $\bar{q} q$-channel, there are no diagrams with two resonant Z-boson propagators present which additionally suppresses the $\gamma \gamma$ channel. The $q \gamma$-channel is even further suppressed by one order of magnitude and entirely negligible.

As an example for a differential cross section, the invariant-mass distribution of the fourlepton system is shown in Fig. 1. The left column focuses on the off-shell sensitive region with its 

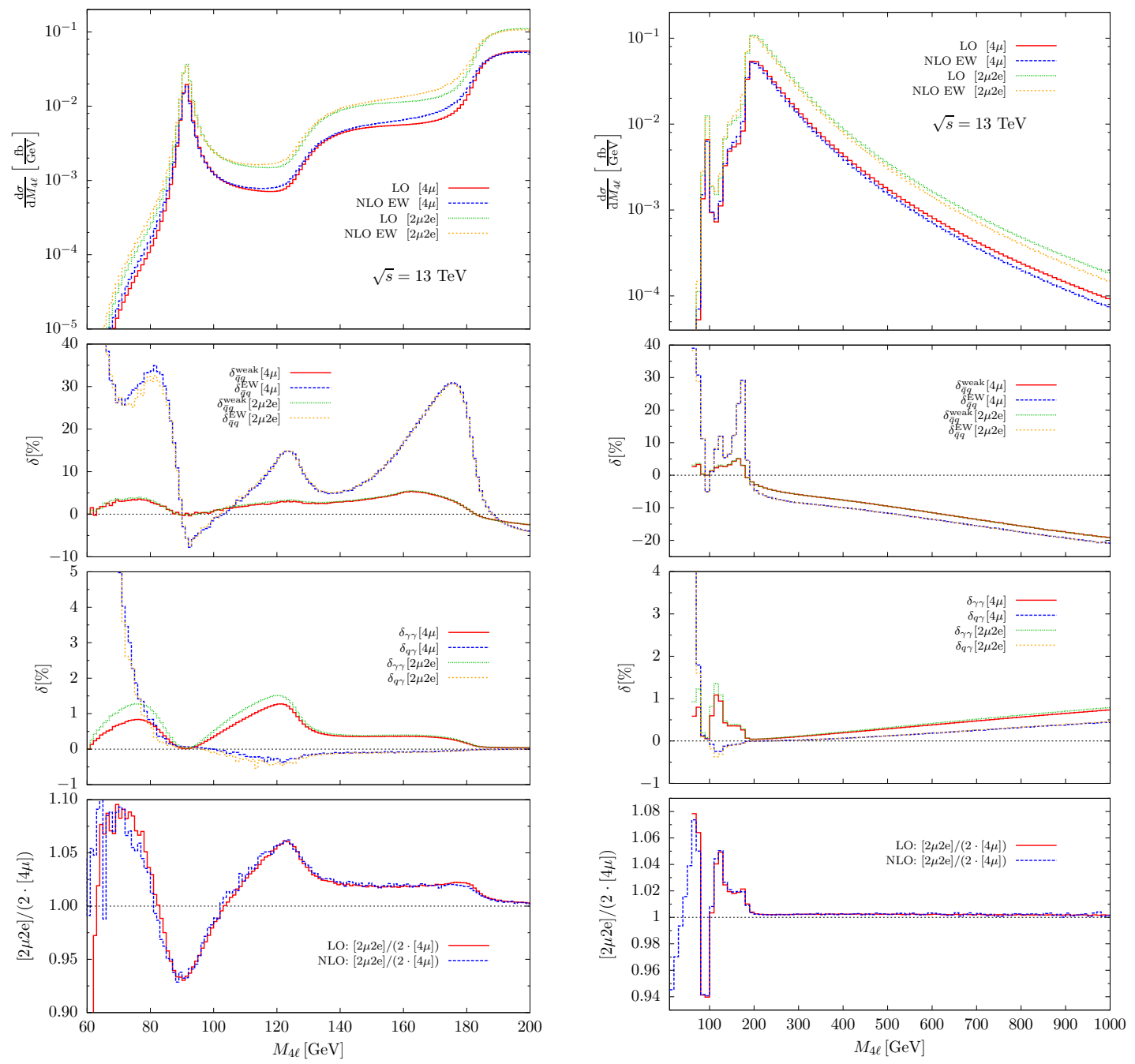

Figure 1: Invariant-mass distribution of the four-lepton system (upper panels), corresponding EW corrections (2nd panels from above), $\gamma \gamma$ and $q \gamma$ contributions (third panels from above) for the unequal-flavour $[2 \mu 2 \mathrm{e}]$ and the equal-flavour $[4 \mu]$ final states in the inclusive setup. The panels at the bottom show the ratio of the $[2 \mu 2 \mathrm{e}]$ and $[4 \mu]$ final states.

resonances and thresholds while the right column illustrates the whole spectrum up to $1 \mathrm{TeV}$. The absolute prediction in the upper row follows the chacteristic pattern of ZZ production: The first peak at $M_{4 \ell}=M_{\mathrm{Z}}$ represents the single Z-boson resonance followed by a decay into four charged leptons ( $s$-channel configuration). The maximum at $M_{4 \ell}=2 M_{\mathrm{Z}}$ represents the pair production threshold where both $\mathrm{Z}$ bosons may be produced on shell ( $t$-channel configuration). The knee above $M_{4 \ell}=M_{\mathrm{Z}}+2 p_{\mathrm{T}, \min } \approx 120 \mathrm{GeV}$ is induced by the kinematical cut of Eq. (2.1) on the lepton transverse momentum.

The panels below the absolute prediction show the relative EW corrections of the $\bar{q} q$ channel and, separately, also the purely weak contribution. The EW corrections, or, more precisely, the photonic corrections, exhibit the typical radiative tails in one-to-one correspondence to the resonance and threshold structure described above: Final state radiation of the real Bremsstrahlung photon 
may shift the value of $M_{4 \ell}$ to lower values and thus shift the location of the resonance enhancement. Since the LO cross section falls off steeply below a resonance or a threshold, the relative correction becomes large and positive, and can reach several tens of percent. Above the ZZ production threshold, the photonic corrections are almost constant with $-2 \%$ to $-3 \%$. In contrast, the purely weak corrections strongly increase in magnitude up to $-20 \%$ at $1 \mathrm{TeV}$. This enhancement is due to electroweak logarithms that become large at high scales. The purely weak corrections become positive below the pair production threshold and stay at the level of $0 \%$ to $+5 \%$ in the off-shell region. This non-trivial sign change is the most remarkable feature of the invariant-mass distribution and illustrates the need for the full off-shell computation of $\mathrm{ZZ}$ production: Because of this sign change it is impossible to include the NLO EW corrections via a global rescaling factor.

The third row of Fig. 1 shows the impact of the photon-induced contributions. The $q \gamma$ contribution is strongly suppressed over the whole spectrum of the distribution, in agreement with the prediction for the fiducial cross section. Above the pair production threshold, the $\gamma \gamma$ channel contributes only at permille level. As the $\gamma \gamma$ contribution is a background contribution with at most one possibly resonant Z-boson propagator, the relative impact increases in the off-shell region up to the percent level. Since this region is dominated by the radiative tails of the photonic corrections, the impact of the $\gamma \gamma$ channel remains small.

The panels in the lowest row display the size of the interfences at differential level via the ratio $\left(\mathrm{d} \sigma_{(\mathrm{N}) \mathrm{LO}}[2 \mu 2 \mathrm{e}] / \mathrm{d} M_{4 \ell}\right) /\left(2 \mathrm{~d} \sigma_{(\mathrm{N}) \mathrm{LO}}[4 \mu] / \mathrm{d} M_{4 \ell}\right)$. Above the pair-production threshold at $M_{4 \ell}=2 M_{\mathrm{Z}}$, the interference is small and constant at the same level as for the fiducial cross section. In the region below $2 M_{\mathrm{Z}}$ where no lepton pair is resonant, the size of the interference effect varies from $-7 \%$ at $M_{4 \ell}=M_{\mathrm{Z}}$ to $+6 \%$ at $M_{4 \ell}=M_{\mathrm{Z}}+2 p_{\mathrm{T}, \min }$. In the region $M_{\mathrm{Z}}+2 p_{\mathrm{T}, \min } \lesssim M_{4 \ell} \lesssim 2 M_{\mathrm{Z}}$, where only one lepton pair can be resonant, the interference effect amounts to $2 \%$. Apart from minor effects at the sub-percent level, the NLO EW corrections do not affect the relative impact of the interferences.

More differential observables on $\mathrm{ZZ}$ production like transverse-momentum distributions, angular correlations, rapidity and invariant-mass distributions have been studied in detail in Ref. [8], including also different event-selection criteria and the role of lepton pairing in the equal-flavour final state.

\section{Conclusion}

In this proceedings contribution we have presented selected results for the theoretical prediction of the production of four charged leptons at next-to-leading order accuracy in the electroweak coupling. The calculation includes all off-shell effects of intermediate virtual photons and massive vector bosons. The purely weak corrections, a gauge invariant subset of the full electroweak corrections, may become large of the order of $-20 \%$ to $-40 \%$ in the high-energy limit due to logarithms of electroweak origin and stay at the level of $-5 \%$ at intermediate scales. The complementary photonic corrections give raise to large radiative tails near resonances or kinematical thresholds of several tens of percent. The photon-induced contributions turn out to be phenomenologically unimportant. Comparing results of the mixed-flavour final state $\mu^{+} \mu^{-} \mathrm{e}^{+} \mathrm{e}^{-}$with those for the equal-flavour final state $\mu^{+} \mu^{-} \mu^{+} \mu^{-}$, we find interference effects in the off-shell sensitive region of the order of $5 \%$. The NLO EW corrections are an important ingredient for precision physics 
within the standard model and its possible extensions, and need to be taken into account in future measurements.

\section{Acknowledgements}

This work was supported by the German Federal Ministry for Education and Research (BMBF) under contract no. $05 \mathrm{H} 15 \mathrm{WWCA} 1$ and by the German Science Foundation (DFG) under reference number DE 623/2-1.

\section{References}

[1] M. Grazzini, S. Kallweit, and D. Rathlev, ZZ production at the LHC: fiducial cross sections and distributions in NNLO QCD, Phys. Lett. B750 (2015) 407-410.

[2] M. Grazzini, S. Kallweit, D. Rathlev and M. Wiesemann, $W^{ \pm} Z$ production at hadron colliders in NNLO QCD, Phys. Lett. B 761 (2016) 179.

[3] M. Grazzini, S. Kallweit, S. Pozzorini, D. Rathlev and M. Wiesemann, $W^{+} W^{-}$production at the LHC: fiducial cross sections and distributions in NNLO QCD, JHEP 1608 (2016) 140.

[4] A. Bierweiler, T. Kasprzik, and J. H. Kühn, Vector-boson pair production at the LHC to $\mathscr{O}\left(\alpha^{3}\right)$ accuracy, JHEP 12 (2013) 071.

[5] J. Baglio, L. D. Ninh, and M. M. Weber, Massive gauge boson pair production at the LHC: a next-to-leading order story, Phys. Rev. D88 (2013) 113005.

[6] B. Biedermann, et al., Next-to-leading-order electroweak corrections to $p p \rightarrow W^{+} W^{-} \rightarrow 4$ leptons at the LHC, JHEP 06 (2016) 065.

[7] B. Biedermann, A. Denner, S. Dittmaier, L. Hofer, and B. Jäger, Electroweak corrections to $p p \rightarrow \mu^{+} \mu^{-} e^{+} e^{-}+X$ at the LHC: a Higgs background study, Phys. Rev. Lett. 116 (2016), no. 16 161803.

[8] B. Biedermann, A. Denner, S. Dittmaier, L. Hofer and B. Jager, Next-to-leading-order electroweak corrections to the production of four charged leptons at the LHC, JHEP 1701 (2017) 033.

[9] S. Kallweit, J. M. Lindert, S. Pozzorini and M. Schonherr, NLO QCD+EW predictions for $2 \ell 2 v$ diboson signatures at the LHC, arXiv:1705.00598 [hep-ph].

[10] S. Catani and M. Seymour, A general algorithm for calculating jet cross-sections in NLO QCD, Nucl.Phys. B485 (1997) 291-419.

[11] S. Dittmaier, A general approach to photon radiation off fermions, Nucl.Phys. $\mathbf{B 5 6 5}$ (2000) 69-122.

[12] S. Actis, A. Denner, L. Hofer, J. N. Lang, A. Scharf and S. Uccirati, RECOLA: REcursive Computation of One-Loop Amplitudes, Comput. Phys. Commun. 214 (2017) 140.

[13] A. Denner, S. Dittmaier and L. Hofer, Collier: a fortran-based Complex One-Loop LIbrary in Extended Regularizations, Comput. Phys. Commun. 212 (2017) 220.

[14] E. Accomando, A. Denner, and C. Meier, Electroweak corrections to $W \gamma$ and $Z \gamma$ production at the LHC, Eur. Phys. J. C47 (2006) 125-146.

[15] A. Denner, S. Dittmaier, M. Roth, and L. Wieders, Electroweak corrections to charged-current $e^{+} e^{-} \rightarrow 4$ fermion processes: Technical details and further results, Nucl.Phys. B724 (2005) 247-294.

[16] NNPDF Collaboration, R. D. Ball, et al., Parton distributions with QED corrections, Nucl. Phys. B877 (2013) 290-320. 